\title{
Synthesis of A Single-Phase Superfine Na-Y Zeolite from Coal Fly Ash
}

\author{
${ }^{\star Z O U ~ L i x i a ~}{ }^{a}$, DING Huiling ${ }^{b}$, ZHENG Lanping $^{b}$, HE Mejuan $^{a}, X$ Q Qiong $^{a}$ \\ a State Key Laboratory Breeding Base of Nuclear Resources and Environment, East China Institute \\ of Technology, Nanchang, 330013, Jiangxi, China \\ b Jiangxi science and technology normal university, Nanchang, Jiangxi, 330000, China \\ aemail: Ixzou1125@163.com
}

Keywords: superfine, zeolite-Y(FAZ), flyash(FA), crystallization, synthesis

\begin{abstract}
The process for the synthesis of flyash-based superfine zeolites-Y (FAZ) were presented, which basically included the alkaline fusion treatment of fly ash (FA), the gels formation by dissolved and aged, following hydrothermal crystallization. A detailed investigation was carried out to determine the effects of conditions of synthesis superfine zeolite-Y. The crystallization structure of zeolite-Y was characterized by X-ray diffraction(XRD) and scanning electron microscope(SEM). The experimental results show that the optimized conditions: Fly ash by alkali fusion(NaOH: fly ash ratio 1.2:1), and dissolved of 60Cusing distilled water and aged of 25C for $22 \mathrm{~h}$. The following, at conditions of $2.2 \mathrm{MNaOH}$ solution, liquid:solid=6.5, the slurry was hydrothermal crystallized at 100C for 24 h. Synthesis of particles exhibits completely grown crystals of zeolites-Y and cubic morphology with approximate dimensions of $400 \mathrm{~mm}$. The crystalinity of zeolite-Y was $88.7 \%$. At the advantage of this paper was to synthesize superfine $\mathrm{Y}$ zeolite that using only flyash as a raw materia and without using any other silicon and aluminum source and any template addition under mild conditions.
\end{abstract}

\section{Introduction}

Coal ash is generated by combustion of coal in a power station as a waste product. It has been established that FA is mainly composed of amorphous material (aluminosilicate glasses) can be converted to zeolites in alkali solutions by hydrothermal treatment. There are many reports for synthesis of zeolite from FA, for example, faujasite, Na-A zeolite, phillipsite and hydroxysodalite. The zeolite-Y has be used in received much attention. It is also reported that use of zeolite-Y can improve catalytic cracking selectivity, reduce coke formation, increase the yield of diesel oil and promote gasoline quality; it can also be used in the fine chemical industry .In the syntheses of zeolite-Y, silica source such as sodium silicate solution and Source of aluminum are currently used and this requires the addition of seeds or initial solution to provide nuclei[1 4] . The aim of this paper was to synthesize a single-phase superfine zeolite-Y from FA. And any source of silicon and aluminum source and any organic template without additional. The products thus obtained were characterized by XRD and SEM. The formation condition and process of a single-phase zeolite-Y are discussed in this article.

\section{Experiment}

\section{Zeolite synthesis}

A homogenous mixture was prepared by proper grinding and mixing of flyash and caustic soda in 1:1.2 ratio. This mixture was heated at about 750Cfor about $2 \mathrm{~h}$. Then , the fusion sample was cooled, milled and mixed thoroughly using distilled water in a breaker. The breaker was kept in a 
water bath and stirred constantly for a few hours. The slurry was subjected to ultrasonic and aging for a few hours. This amorphous alumino-silicate gel was formed. This gel was then subjected to crystallization between 40C 100Cfor about 10 30 h in specially designed stainless alloy autoclaves with thin walls which allow a fast heat transfer. The solid crystalline product was recovered by filtration and washed with distilled-deionized water thoroughly until the filtrate $\mathrm{pH}$ was 11 , and dried at $80^{\circ} \mathrm{C}$ for $24 \mathrm{~h}$ in an air oven.

\section{Characterization.}

The materials obtained were characterized by various conventional methods. Powder X-ray diffraction (XRD) patterns were taken on a Rigaku X-ray diffractometer using Ni-filtered Cu KR radiation $(30 \mathrm{kV}, 16 \mathrm{~mA})$. Particle morphology was observed by an Hitachi scanning electron microscope(SEM).

\section{Results and Discussion}

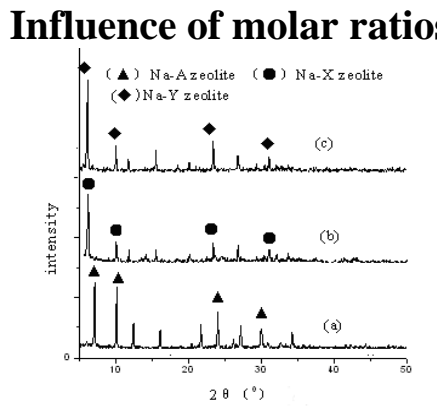

Fig. 1. Influence of molar ratio $\mathrm{SiO}_{2} / \mathrm{Al}_{2} \mathrm{O}_{3}$ :

(a) 0.9, (b) 2.0, (c)pure FA
Fig. 2 Influence of aging temperatures :

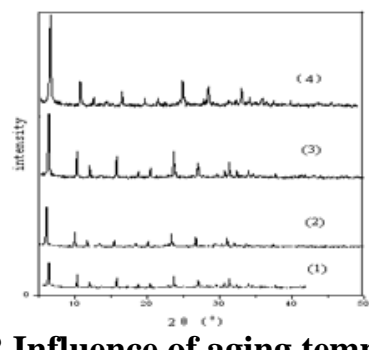

(1) $80 \mathrm{C}$, (2) $50 \mathrm{C}$ and(3) $40 \mathrm{Cfor} 24 \mathrm{~h}$; (4) at $60 \mathrm{C}$ for $2 \mathrm{~h}$ and $25 \mathrm{C}$ for $22 \mathrm{~h}$

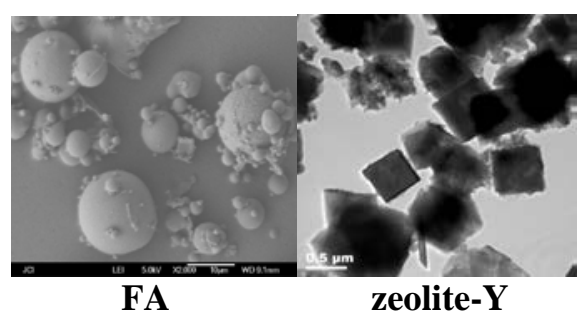

Fig. 4 SEM spectrum of zeolite-Y

Figure 1 shows the XRD patterns of the synthesized samples were investigated by changing other $\mathrm{SiO}_{2} / \mathrm{Al}_{2} \mathrm{O}_{3}$ ratio from 0.9 to 3.5 and the crystallization temperature at $100 \mathrm{C}$. The patterns of the material formed at $\mathrm{SiO}_{2} / \mathrm{Al}_{2} \mathrm{O}_{3}=0.9$ possesses many diffraction peaks, which can be assignable to zeolite-A . When the ratio of $\mathrm{SiO}_{2} / \mathrm{Al}_{2} \mathrm{O}_{3}$ increase, new peaks of faujasite appear (pattern (b)-(c)). The synthetic faujasite type zeolite takes in two forms with the same crystal structure at different $\mathrm{SiO}_{2} / \mathrm{Al}_{2} \mathrm{O}_{3}$ molar ratio. One was zeolite-X with $\mathrm{SiO}_{2} / \mathrm{Al}_{2} \mathrm{O}_{3}=2.0$ and another was zeolite-Y using only the starting materials flyash $\left(\mathrm{SiO}_{2}\right.$ and $\mathrm{Al}_{2} \mathrm{O}_{3}$ ratio of 2.5 3.5 about).

\section{Influence of aging conditions}

It has been established that the amorphous aluminosilicate in FA is easier to dissolve than the crystalline one such as R-quartz and mullite in alkali solutions. To clarify the detailed formation process of zeolite-Y, effect on the final product of crystallization were characterized under different aging conditions. In Figure 2, as can be seen, the zeolite-Y was synthesised ,but the crystallization strength reduction with temperature rise. This is because the silicon aluminum dissolved easily formation gel under high temperature, deposited on the surface of FA, further influence in which the silicon aluminum dissolved, resultd crystallization decreased. The intensity of XRD peak of the sample-(4) was highest which that it was dissolved of 60Cand aged of 25Cfor 22h. So, it was used as a fixed aging condition in the following synthesis experiments.

\section{Influence of the conditions of crystallization}

Fig. 3(1) illustrates the XRD patterns of three samples synthesized from FA at the condition of 1 $\mathrm{M}, 2.2 \mathrm{M}$ and $3 \mathrm{M} \mathrm{NaOH}$ solution and crystallization for $24 \mathrm{~h}$. According to XRD patterns of three samples obtained, only sample (2.2 $\mathrm{MNaOH}$ solution) was confirmed by pure-form zeolite-Y without the formation of other types of zeolites. Hui and Chao pointed out that synthesis of zeolite Y depends on the rate of gel dissolution[5], the number and distribution of nuclei in prepared initial 
gel, and the crystal growth rate during hydrothermal treatment. The structural formation of zeolite $\mathrm{Y}$ may be explained in turn depend on other factors such as synthesis temperature, crystallization time, composition molar ratios of initial gel, etc.
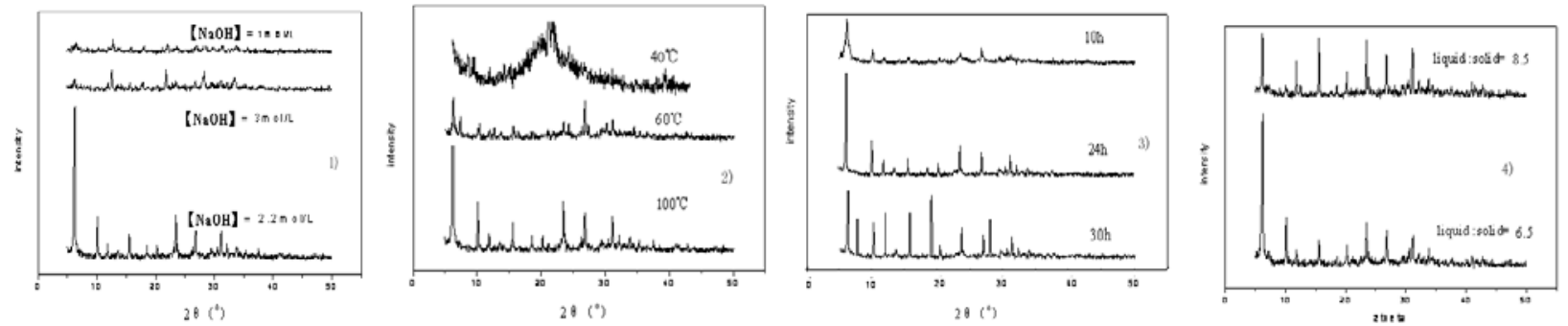

Fig. 3 XRD patterns of FAY in differentat contidions of1) alkalinity concentration, 2) crystallization time,

3 ) crystallization temperatures, 4) the ratio of liquid to solid

The alumino-silicate fused mass gel obtained is amorphous and changes to the crystalline state when subjected to hydrothermal crystallization. The results presented in Fig.3(2,3) reveals that crystallization temperature and time influences zeolitic crystallinity. Percent crystallinity of zeolite-Y increases significantly until up to 100Cfor $24 \mathrm{~h}$. The crystallization time increases to 30h, the other peaks to formed. Therefore, crystallization contidions identified as $100 \mathrm{Cfor} 24 \mathrm{~h}$ in the experiment.

Fig. 3(4) shows the XRD patterns of samples produced using different molar ratios of liquid to solid under the same synthetic conditions. The XRD patterns indicate that all samples exhibit a zeolite-Y structure. Notably, the samples-2(liquid:solid=6.5) show significantly higher broadened diffraction peaks, with higher yield of crystallization. The relative crystalinities calculated[6] for samples -1 (liquid:solid=8.5)and samples -2 are found to be $74.7 \%$ and $88.7 \%$, respectively.

\section{Morphological studies}

The SEM photographs in Fig.3 depict the sample transformation of FA into Zeolite-Y. Samples zeolite-Y are fairly uniform in crystal size, and the individual aggregates are composed of closely packed nanocrystals, and have smaller average aggregate sizes. This is probably because less uniform crystallization occurred in gel. For the sample the average particle (aggregate) size was estimated from at least $400 \mathrm{~nm}$ particles in the SEM images.

\section{Conclusions}

Synthesis of FAZ is directly related to extraction of silicates and aluminates form FA using sodium hydroxide. From the information presented in this paper, $\mathrm{Si}^{4+}$ and $\mathrm{Al}^{3+}$ ions are eluted from fusing FA by dissolution of amorphous material during the aging and crystallization to form a single-phase Y type zeolite in the autoclave . The Y type zeolite were characterization by XRD and SEM patterns, the following conclusions can be drawn.

A homogenous fusion mixture was prepared by proper grinding and mixing of FA and caustic soda in 1:1.2 ratio. This mixture was calcined at about 750Cfor about $2 \mathrm{~h}$. Then sample was dissolved at 60Cand aged at 25Cfor 22h, to be gel formation. The gel and water as the ratio of liquid to solid is 6.5 , in $2.2 \mathrm{M} \mathrm{NaOH}$ solution to crystallization for $24 \mathrm{~h}$ at $100 \mathrm{C}$ using the autoclave. The maximum purity of zeolite -Y was obtained, crystalinity calculated for samples was $88.7 \%$. SO, the $\mathrm{SiO}_{2}$ and $\mathrm{Al}_{2} \mathrm{O}_{3}$ ratio of 2.5 3.5 in FA favours formation of Zeolite- $Y$. On modification of the actual $\mathrm{SiO}_{2}$ and $\mathrm{Al}_{2} \mathrm{O}_{3}$ ratio, by increasing sodium silicate or sodium aluminate content, it was possible to synthesise zeolite- $\mathrm{X}$ or zeolite- $\mathrm{A}$ at $\mathrm{SiO}_{2} / \mathrm{Al}_{2} \mathrm{O}_{3}$ of 2.0 or 0.9 respectively . The important feature of this novel procedure is to produce superfine zeolite-Y and crystalinity $88.7 \%$ in one vessel without using any other silicon and aluminum source and any template addition. The high volume utilization of FA for zeolite production technology compared to other mediumr low value utilization, this production technology has advantages of value addition, offering an edge over 
other flyash utilisation technologies currently used.

\section{Acknowledgment}

This study was supported in part by Jiangxi Provincial Department of science and technology support program in china(20122BBG70084-2). The help and cooperation rendered by the management of Fengcheng Thermal Power Station in collecting flyash for this research work is greatly acknowledged.

\section{References}

[1] Rayalu S, Meshram S U, Hasan M Z. Highly crystalline faujasitic zeolites from flyash. Journal of Hazardous Materials, B, Vol.77(2000),P.123-131

[2] Juliana C I, Denise A F, Fernando S S, ect. . Characteristics of Brazilian coal fly ashes and their synthesized zeolites Original Research . Article.Fuel Processing Technology, Vol.97(2012),P.38-44

[3] Belviso C, Cavalcante F, Javier Huertas F, ect.. The crystallisation of zeolite (X- and A-type) from fly ash at $25{ }^{\circ} \mathrm{C}$ in artificial sea water Original Research Article. Microporous and Mesoporous Materials, Vol.162(2012), P.115-121

[4] Kazemian H, Naghdali Z, Ghaffari Kashani T, ect..Conversion of high silicon fly ash to Na-P1 zeolite: Alkaline fusion followed by hydrothermal crystallization Original Research Article. Advanced Powder Technology, Vol.21(2010), P. 279-283

[5] Claudia B, Francesco C, Saverio F. Synthesis of zeolite from Italian coal fly ash: Differences in crystallization temperature using seawater instead of distilled water Original Research Article. Waste Management ,Vol.30(2010), P. 839-847

[6] Hui K S, Chao C Y H. Effects of step-change of synthesis temperature on synthesis of zeolite 4A from coal fly ash, Micropor. Mesopor. Mater. Vol.88 (2006),P.145-151

[7] Fotovat F , Kazemian H, Kazemeini M. Synthesis of Na-A and faujasitic zeolites from high silicon fly ash. Materials Research Bulletin. Materials Research Bulletin,Vol.44 (2009),P.913-917 